\title{
Spatial ultrasonic cleaning process control based on its current state evaluation
}

\author{
Volodymyr Morkun ${ }^{1}$, and Olha Kravchenko ${ }^{1, *}$ \\ ${ }^{1}$ Kryvyi Rih National University, 11 Vitalii Matusevych Str., Kryvyi Rih, 50027, Ukraine
}

\begin{abstract}
Ultrasonic cleaning is one of the most promising types of cleaning in terms of environmental friendliness, cost and efficiency. The condition of the cleaning body must be taken into account for optimal control of the ultrasonic cleaning process. This allows you to irradiate only those areas that really need it. The modelling of the process of ultrasonic cleaning of bodies of different configurations and the analysis of the parameters of ultrasonic responses at different stages of cleaning were performed. This allowed us to identify the parameters by which the assessment of the process should be formed. The main parameter was the change in the time of receipt of the threshold value of the signal, and the auxiliary - the change of the nonlinearity coefficient of the second order. The change in the time of receipt of the threshold value of the signal is an indicator of dirt peeling, and the change in the nonlinearity coefficient demonstrates the approach to the final result of cleaning. These parameters became clear input data for the 3-D fuzzy interval controller. The functions of affiliation were defined and the base of rules was formed. Modelling of the ultrasonic cleaning process using the established method of estimating the course of the process and the use of 3-D fuzzy interval controller showed about $35 \%$ energy savings.
\end{abstract}

\section{Introduction}

Effective cleaning is an important component of both production cycles and the possibility of ongoing equipment repairs. High quality in the absence of heavy manual labour and the use of aggressive chemicals makes ultrasonic cleaning one of the most promising types of cleaning. Modern technological developments and general trends in energy saving pose new challenges to increasing energy efficiency of ultrasonic cleaning. There are several approaches to overcoming these challenges. First, it is a study of the features of physical processes that provide the process of ultrasonic cleaning. The main physical phenomenon that provides ultrasonic cleaning is ultrasonic cavitation - the implosion of bubbles that occur under the action of changes in fluid pressure during sonication. This is the release of energy, which separates the dirt from the body. Cavitation in the ultrasonic bath has a zonal distribution, so the cleaning efficiency is affected not only by the parameters of the ultrasound, but also the size of the bath, the location of the emitters. Adjusting these parameters can significantly improve the quality of cleaning, as demonstrated in [1]. The distribution of cavitation is also greatly influenced by the frequency of ultrasound, and other parameters, such as fluid temperature, ultrasound intensity, oxygen content, affect the power of cavitation [2]. The large number of interrelated parameters that affect the ultrasonic cleaning process complicate the process of forming a control based on standard methods. Therefore, another approach to increase the energy efficiency of ultrasonic cleaning is to improve the methods of controlling this process. Thus, Duran Fecir proposed to use the evaluation of the process by the physical properties of the cleaning fluid, namely: conductivity and turbidity [3]. In [4], the evaluation of the process is based on the temperature of the liquid. Such approaches increase the efficiency of ultrasonic cleaning, but ignore the real state of the cleaning body, forming a control based on indirect evaluation.

More flexible is the formation of assessment based on machine learning, which was used in [5] and [6]. In these works, the condition of the treatment facility (in both cases, part of the pipe) was assessed by applying a neural network to the received ultrasound responses. The results of these experiments prove the possibility of using the analysis of ultrasonic responses to assess the degree of contamination of the body, but the algorithms used are limited by the shape of the treatment object and cannot be applied in the general case. Therefore, it is necessary to develop an evaluation methodology that will allow you to form a control based on the state of the treatment plant of any configuration.

For greater efficiency, the control of the ultrasonic cleaning process should take into account its spatial distribution and the actual state of the cleaning body. Otherwise, there will be ultrasonic treatment of already cleaned areas or insonation with insufficiently intensive ultrasound of areas that require it. For optimal redistribution of the intensity of each emitter of the ultrasonic bath, it is proposed to use a 3-D fuzzy interval controller type 2 [7], which is essentially designed for

\footnotetext{
* Corresponding author: kravchenko@knu.edu.ua
} 
spatially distributed processes with complex expert evaluation of input parameters.

Modeling the application of a new method for assessing the state of the ultrasonic process and the formation of control using a 3-D fuzzy interval controller type 2 showed an increase in energy efficiency of cleaning by $40 \%$.

\section{Problem description}

Let the ultrasonic cleaning take place with the help of $\mathrm{m}$ ultrasonic emitters located in the given positions and given by the intensity coefficients $u(z)=$ $\left(u\left(z_{1}\right), u\left(z_{2}\right), \ldots, u\left(z_{m}\right)\right)$. Input parameters are measured in $P$ spatial positions $Z_{1}, Z_{2}, \ldots Z_{p} . P$ and $m$ are independent. With the help of spectral analysis of ultrasonic responses, it is necessary to form a method for assessing the course of the ultrasonic cleaning process, followed by the formation of a spatially distributed control action. Carry out comparative modeling of control on the basis of the developed technique concerning traditional control of process of ultrasonic cleaning limited by a time indicator.

\section{Methods for assessing the state of the course of cleaning by ultrasonic responses in the tank}

\subsection{Evaluation of mechanical properties of the body using ultrasound nonlinearity}

Ultrasonic non-destructive evaluation is widely used to quantify the mechanical and structural properties of the body. It has established itself well in many industries and continues to expand its application [8-10]. Existing studies indicate the feasibility of using to determine the degree of heterogeneity of the mechanical properties of the body coefficients of nonlinearity of the second and third orders $[9,11-12]$

$$
\begin{gathered}
\boldsymbol{\beta}_{2}=\frac{\boldsymbol{A}_{\mathbf{2}}}{\boldsymbol{A}_{\mathbf{1}}^{\mathbf{2}}}, \\
\beta_{3}=\frac{A_{3}}{A_{1}^{3}} .
\end{gathered}
$$

But in the case of ultrasonic cleaning, the situation is complicated by a significant change not only in the properties of the body, but also in the properties of the liquid, because some of the contaminated particles will be in a suspended state after exfoliation.

In the general case, the loss of signal intensity is described by an exponential dependence

$$
I(x)=I_{0} e^{-2 \alpha x},
$$

where $I_{0}$ is the intensity of the sound wave at the entrance to the material, $\alpha$ is the value of the attenuation coefficient, which depends on the frequency of the ultrasonic wave and the properties of the material.
In addition, the loss of intensity will depend on the ratio of the densities of the boundary media and the speed of ultrasound in them. With this in mind, the intensity loss can be written as

$$
I(x)=I_{0} e^{-2 \alpha x}\left(\frac{\rho_{2} \mathrm{c}_{2}-\rho_{1} \mathrm{c}_{1}}{\rho_{2} \mathrm{c}_{2}+\rho_{1} \mathrm{c}_{1}}\right)^{2},
$$

where $\rho_{1}, \mathrm{c}_{1}, \rho_{2}, \mathrm{c}_{2}$ are body density and ultrasound rate for 1 and 2 media, respectively.

If we take into account the absorption and scattering of suspended particles of contaminants in the substance [13], we obtain the following general view for the reflected intensity, which will be returned to the sensor when the reflected ultrasonic wave from the body

$$
\begin{gathered}
I(x)=I_{0} e^{-\sqrt{n}(1-\eta)} e^{-2 \alpha x}\left(\frac{\rho_{2} c_{2}-\rho_{1} c_{1}}{\rho_{2} c_{2}+\rho_{1} c_{1}}\right) e^{-\sqrt{n}(1-\eta)}= \\
=I_{0} e^{-2 \alpha x}\left(\frac{\rho_{2} c_{2}-\rho_{1} c_{1}}{\rho_{2} c_{2}+\rho_{1} c_{1}}\right) e^{-2 \sqrt{n}(1-\eta)}= \\
=I_{0} e^{-2(\alpha x+\sqrt{n}(1-\eta))\left(\frac{\rho_{2} c_{2}-\rho_{1} c_{1}}{\rho_{2} c_{2}+\rho_{1} c_{1}}\right)}
\end{gathered}
$$

where $\eta=\int_{0}^{\infty} \exp \left\{-\frac{1}{V} \sigma(\lambda, r) Z\right\} \varphi(r) d r$ is concentration by size; $\varphi(r)$ is particle size distribution function, $V=\frac{\pi d^{2}}{4} Z$ - where $d$ is the diameter of the emitter, $z$ is the distance to the body, $\bar{n}$ is the average concentration of solid particles in the liquid.

According to formula (5) we obtain the largest change in the value coming to the sensor when changing the ratio at the boundary of the media, ie with complete purification. The change in intensity also significantly depends on the thickness of the contamination, the concentration of contamination in the liquid and the particle size distribution.

Also during cleaning there is a change in the configuration of the body, which also affects the parameters of the ultrasonic signal received by the sensor. To determine the influence of the features of the body configuration on the time of receipt of the threshold value and the nonlinearity coefficients of the second and third orders, modelling was performed for different shapes of the body and the corresponding values were calculated..

\subsection{Modelling of ultrasonic cleaning bodies of different configurations}

$\mathrm{K}$-wave was chosen as the simulation software. The advantage of this product is the simulation of large-scale propagation of ultrasonic waves in a reasonable time [1415]. The sensor and the emitter were located in the lower right corner of the simulated area. The section of the cleaning body was set with a density $\rho=7800 \mathrm{~kg} / \mathrm{m}^{3}$ and the speed of propagation of the ultrasonic wave $\mathrm{c}=5100$ $\mathrm{m} / \mathrm{s}$, which corresponds to the metal product. The contamination was determined by the density $\rho=3100 \mathrm{~kg}$ $/ \mathrm{m}^{3}$ and the speed of propagation of the ultrasonic wave 
$\mathrm{c}=2500 \mathrm{~m} / \mathrm{s}$, which corresponds to corrosion. The following were chosen as different shapes of areas: the sector of a circle, the sector of asteroids and the sectors of a circle and asteroids with holes. Ultrasonic responses were recorded for the body of each configuration and with different thickness of contamination. These responses were processed using a fast Fourier transform and obtained the values of the largest 3 amplitudes (Table 1).

Table 1. Parameters of ultrasonic responses when cleaning bodies of different configurations

\begin{tabular}{|c|c|c|c|c|c|c|c|c|}
\hline $\begin{array}{l}\text { Body } \\
\text { number }\end{array}$ & $\begin{array}{l}\text { The initial } \\
\text { appearance of } \\
\text { the body with }\end{array}$ & $\begin{array}{l}\text { The thickness of } \\
\text { the } \\
\text { contamination, } \mathrm{mm}\end{array}$ & $\begin{array}{l}\text { Signal } \\
\text { time, } \mu \mathrm{s}\end{array}$ & \multicolumn{3}{|c|}{$\begin{array}{l}\text { The value of the } \\
\text { amplitudes of the first } \\
\text { three harmonics, } \mathrm{Pa}\end{array}$} & \multirow{2}{*}{$\begin{array}{l}\beta_{2} \\
1.339 \\
\end{array}$} & \multirow{2}{*}{$\begin{array}{c}\beta_{3} \\
3.053\end{array}$} \\
\hline \multirow[t]{4}{*}{1} & & 60 & 2669 & 0.2379 & 0.0758 & 0.0411 & & \\
\hline & & 40 & 2818 & 0.2922 & 0.1046 & 0.0612 & 1.225 & 2.453 \\
\hline & & 20 & 2956 & 0.3173 & 0.1004 & 0.0569 & 0.997 & 1.781 \\
\hline & & 0 & 3105 & 0.36 & 0.084 & 0.0833 & 0.648 & 1.785 \\
\hline \multirow[t]{4}{*}{2} & & 60 & 2467 & 0.1026 & 0.0229 & 0.0090 & 2.175 & 8.333 \\
\hline & & 40 & 2728 & 0.154 & 0.0633 & 0.0199 & 2.669 & 5.449 \\
\hline & & 20 & 2884 & 0.1498 & 0.0619 & 0.0038 & 2.758 & 1.13 \\
\hline & & 0 & 2914 & 0.1104 & 0.03 & 0.0059 & 2.461 & 4.385 \\
\hline \multirow[t]{5}{*}{3} & & 60 & 2466 & 0.1016 & 0.0238 & 0.0099 & 2.306 & 9.44 \\
\hline & & 40 & 2728 & 0.1601 & 0.0571 & 0.0206 & 2.228 & 5.02 \\
\hline & & 20 & 2882 & 0.1414 & 0.0599 & 0.0076 & 2.996 & 2.688 \\
\hline & & 0 & 2924 & 0.0927 & 0.025 & 0.01 & 2.909 & 12.553 \\
\hline & & $\begin{array}{l}\text { the cleaned } \\
\text { opening }\end{array}$ & 3033 & 0.0846 & 0.0314 & 0.0158 & 4.387 & 26.094 \\
\hline \multirow[t]{5}{*}{4} & & 60 & 2669 & 0.2377 & 0.0759 & 0.0409 & 1.343 & 3.045 \\
\hline & & 40 & 2818 & 0.2942 & 0.1041 & 0.0625 & 1.203 & 2.454 \\
\hline & & 20 & 2956 & 0.3238 & 0.101 & 0.06 & 0.963 & 1.767 \\
\hline & & 0 & 3106 & 0.3419 & 0.0718 & 0.0831 & 0.614 & 2.079 \\
\hline & & $\begin{array}{l}\text { the cleaned } \\
\text { opening }\end{array}$ & 3105 & 0.3629 & 0.0869 & 0.0824 & 0.66 & 1.724 \\
\hline
\end{tabular}

For clarity, we construct graphs of dependences (Fig. 2), which show that the presence of a cleaned hole causes an increase in the nonlinear component (secondorder coefficient) regardless of the geometric shape of the body.

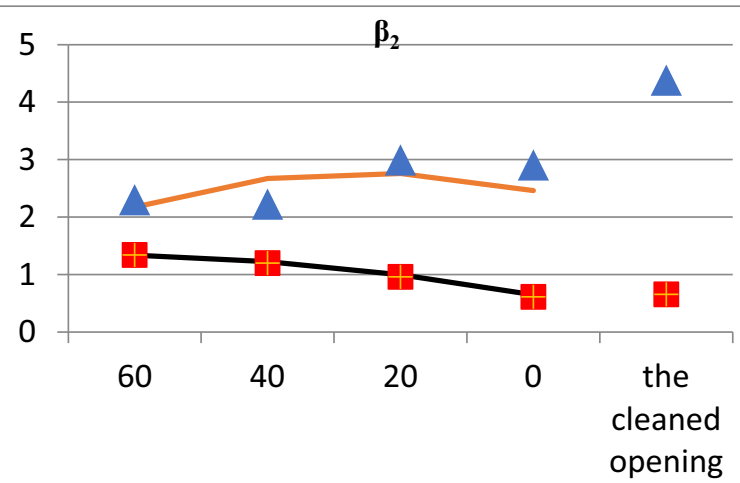

$-1-2 \Delta 3 \pm 4$

Fig. 1. Nonlinearity coefficient of second order with different thickness pollution.

For the sector range - significantly (from 2.909 to 4.387 ) for astroyidalnoho sector small (0.614 to 0.66$)$. The general nature of the change is uneven - reduction coefficient $\beta_{2}$ occurs when a layer of pollution below a certain level, and the increase can be observed for certain geometric shape when the layer of pollution reduced, but overall remains high. In Fig. 2 shows that for any body $\beta_{2}$ factor value at the last stage of cleaning is reduced.

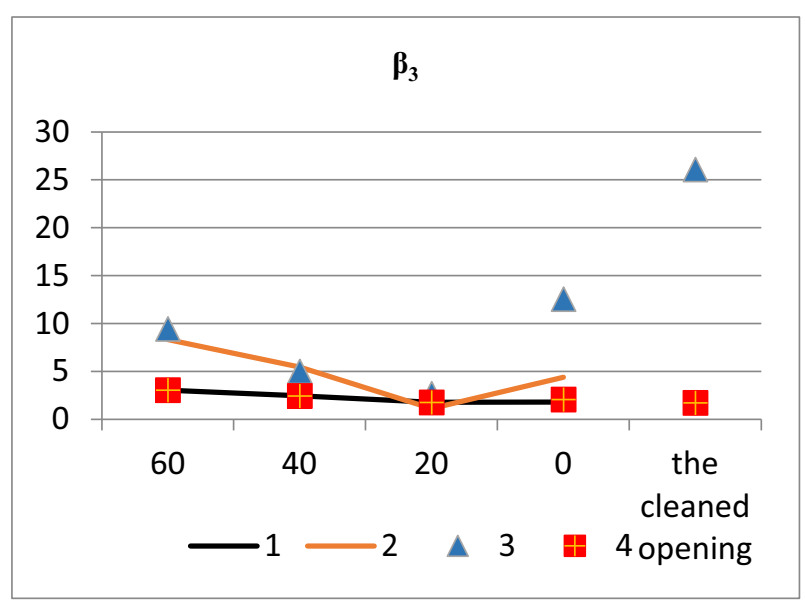

Fig. 2. Nonlinearity coefficient of third order with different thickness pollution.

Cleaning the holes, on the contrary, causes it to increase. Therefore, we can draw the following conclusion: an increase in the coefficient $\beta_{2}$ indicates either the cleaning of the holes, or a decrease in 
contamination, the layer of which remains quite significant.

The nonlinearity coefficient of the third order is even more dependent on the geometry of the body and does not show patterns in the process of ultrasonic cleaning. Therefore, the decision was limited to the analysis of only the second-order coefficient.

An important factor that has a stable behavior and allows you to assess the course of the process of ultrasonic cleaning is the time after which the threshold value of the signal. In fig. 8 shows that the change in time occurs almost linearly with a constant increase. Nonlinearity is observed only at the stage of hole cleaning, when the change in the time of receipt of the threshold signal may decrease.

With the increase of suspended particles of contamination in the liquid, the state of the liquid, not the body, has a greater influence on the response signal. There was a decrease in the time of receipt of the threshold signal and an increase in the main amplitude.

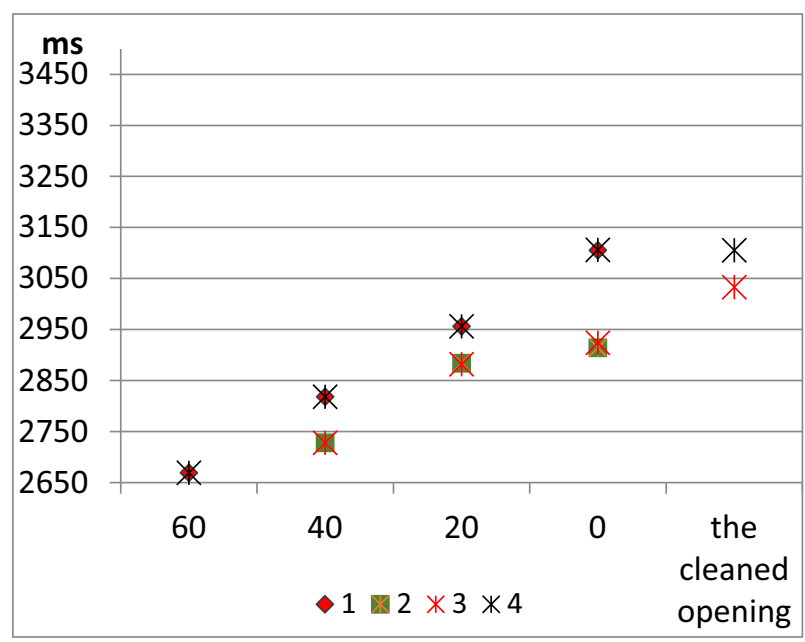

Fig. 3. The time of receipt of the threshold value of the signal depending on the thickness of the contamination for table 1 bodies.

Thus, the value of the change in the time of the signal, the coefficient of nonlinearity of the second order and the value of the change of the main amplitude, you can estimate the state of the ultrasonic cleaning, namely:

1. The change in the thickness of the contamination almost directly proportionally affects the change in the time of receipt of the ultrasonic response.

2. The coefficient of nonlinearity of the second order can determine the thickness of the contamination: a significant increase in the coefficient (more than 50\%: $\frac{|4.387-2.909|}{2.909}=0.51$ for body 3 in table 2) with increasing time of receipt indicates the cleaning of various holes, ie the final stage of purification. The main amplitude decreases.

3. A less significant increase in the nonlinearity coefficient of the second order with increasing time of receipt $\left(\frac{|2.996-2.228|}{2.228}=0.5\right.$ for the body 3$)$ is evidence that the contamination is still quite significant and the area requires intensive cleaning. The behavior of the main amplitude is arbitrary.
4. The increase of the main amplitude with the decrease of the nonlinearity coefficient of the second order is a clear indicator of a stable purification process.

\section{Control of ultrasonic cleaning based on 3-D interval fuzzy controller}

\subsection{Controller inputs}

Therefore, the input parameters that will allow to assess the state of the ultrasonic cleaning process are the last two measured values of the time of receipt of the threshold signal and the nonlinearity coefficient of the second order.

The value of the "change" of the signal is obtained as the relative difference of the last two measurements of the threshold time. If this value is equal to 0 , then the product of the ratios of the nonlinearity coefficients of the 2nd and 3rd orders is chosen as this parameter

$$
x_{1}=\frac{t_{2}-t_{1}}{t_{2}}
$$

where $t_{2}, t_{1}$ are the times of receipt of the threshold value of the signal for the current and past measurements.

To determine the "purity" of the signal, we use the ratio of nonlinearity coefficients of the 2 nd order

$$
\mathrm{x}_{2}=\frac{\beta_{2}^{(2)}-\beta_{2}^{(1)}}{\beta_{2}^{(2)}},
$$

where $\beta_{2}^{(2)}, \beta_{2}^{(1)}$ are values of the current and past nonlinearity coefficients of the 2 nd order.

\subsection{Membership function for inputs}

The values of $x_{1}, x_{2}$ are normalized in the interval [-1,1]. Since the sign of the input parameter is fundamental, the normalization was not carried out evenly, but as the ratio of the found value to the maximum modulo with the same sign. For both input variables, we set five linguistic labels: significant positive (LP), mean positive (MP), zero $(\mathrm{Z})$, mean negative $(\mathrm{MN})$, and significant negative (LN). Given that the current value of the signal received by this sensor is most affected by the state of the immediate environment, the following type was chosen for the membership functions

$$
\begin{aligned}
& \underline{\mu}\left(x_{i}, z\right)=\exp \left(-\frac{\left(\left(x_{i}-a\right)^{2}+2\left(|2| z-z_{0}|-1|-1\right)^{2}\right)}{\sigma_{1}}\right) \\
& \bar{\mu}\left(x_{i}, z\right)=\exp \left(-\frac{\left(\left(x_{i}-a\right)^{2}+2\left(|2| z-z_{0}|-1|-1\right)^{2}\right)}{\sigma_{2}}\right)
\end{aligned}
$$

where $\mu\left(x_{i}, z\right)$ та $\bar{\mu}\left(x_{i}, z\right)$ are the upper and lower values of the membership function, $x_{i}$ is the crisp input, $z=$ 
$\left(z_{1}, z_{2}, \ldots, z_{P}\right)$ is the radial input coordinate (the sensor location), $a$ is the value of the linguistic mark with which the membership function acquires the maximum value: for (LP) $-a=1$, for (MP) $-a=0.5$, for (Z) $-a=0$, for $(\mathrm{MN})-\mathrm{a}=-0.5$ and for $(\mathrm{LN})-\mathrm{a}=-1, z_{0}$ is the value of the spacious radial output coordinate (radiator location), $\sigma_{1}$ та $\sigma_{2}$ - are parameters conditioned by physical properties of the cleaning tank and the number of sensors and outputs and belong to the interval $[0,1], i=1,2$ is the number of variables. Fig. 4 shows a diagram for the linguistic mark $(\mathrm{MN})$ and the output $z_{0}=0.2$ with $\sigma_{1}=$ 0.1 and $\sigma_{2}=0.2$.

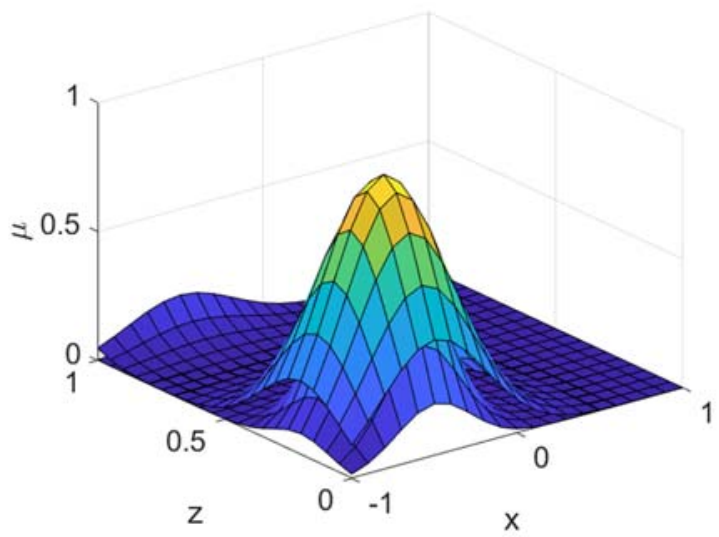

Fig. 4. Diagrams of the upper and lower membership functions for the mean positive mark $(\mathrm{MN})$ for the output $\mathrm{z}_{0}=0.2$;

This type of function will provide a spatial effect of the sensor signal on all emitters. At the minimum distance we get the maximum value of the function, and at the distance of the output from the input the value of the function decreases.

\subsection{The rule base}

Taking into account the above-described method of evaluating the course of the ultrasonic cleaning process, a base of rules for a fuzzy controller was formed (Table 2). This database is two-dimensional and does not depend on the number of sensors. Spatial distribution is taken into account through the membership functions of the input parameters. When forming the database of rules, the main role is taken into account the time of receipt of the threshold value of the signal $\left(x_{1}\right)$.

Table 2. The rule base

\begin{tabular}{|c|c|c|c|c|c|}
\hline $\boldsymbol{x}_{\mathbf{2}} / \boldsymbol{x}_{\mathbf{1}}$ & $\widetilde{\boldsymbol{L N}}$ & $\widetilde{\boldsymbol{M N}}$ & $\widetilde{\boldsymbol{Z}}$ & $\widetilde{\boldsymbol{M P}}$ & $\widetilde{\boldsymbol{L P}}$ \\
\hline$\widetilde{\boldsymbol{L N}}$ & $\tilde{S}$ & $\widetilde{M}$ & $\widetilde{M}$ & $\tilde{L}$ & $\tilde{L}$ \\
\hline$\widetilde{\boldsymbol{M N}}$ & $\tilde{S}$ & $\widetilde{M}$ & $S$ & $\widetilde{M}$ & $\tilde{L}$ \\
\hline$\widetilde{\boldsymbol{Z}}$ & $\tilde{S}$ & $\tilde{S}$ & $\tilde{S}$ & $\widetilde{M}$ & $\widetilde{L}$ \\
\hline$\widetilde{\boldsymbol{M P}}$ & $\tilde{S}$ & $\tilde{S}$ & $\tilde{S}$ & $\widetilde{M}$ & $\tilde{L}$ \\
\hline$\widetilde{\boldsymbol{L P}}$ & $\tilde{S}$ & $\tilde{S}$ & $\widetilde{M}$ & $\tilde{L}$ & $\tilde{L}$ \\
\hline
\end{tabular}

A negative value of this factor indicates contamination of the liquid, and a positive value indicates the peeling of the contamination.

For the second parameter, which is a relative change in the nonlinearity coefficient of the second order $\left(x_{2}\right)$, the following dependences: a negative value indicates that the purification is occurring and is approaching the final stage, and a positive value indicates the presence of a significant amount of contamination.

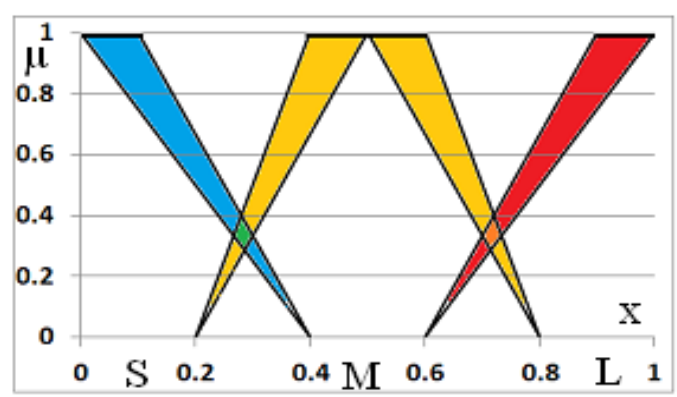

Fig. 5. Membership function for output.

The original membership function is twodimensional and determines the value for each emitter separately. The output function is normalized in the interval $[0,1]$, because the clear value of the output - the intensity of the emitter - is always non-negative. The second view is shown in Fig. 5.

\subsection{Algorithm of the controller based on 3-D fuzzy interval set type 2}

According to work [7], the operation of the controller based on the 3-D fuzzy interval set of type 2 will occur in the following steps:

1. Obtaining a clear input according to the method described in this article and its phasing.

2. Formation of the conclusion on the basis of base of rules.

3. Combining spatial information and reducing it with a maximum operation.

3. Data accumulation and type reduction through the search for a centroid by the Karnik-Mendel algorithm [16].

4. Formation of a clear conclusion.

\section{Results and discussion}

To confirm the effectiveness of the newly developed method for evaluating the course of the ultrasonic cleaning process, a complete modelling of the process was performed using a 3-D fuzzy interval controller. The location of the contaminated body, 4 emitters and 4 sensors were specified (Fig. 6). The figure shows working emitters in the form of concentrated circles purification cycle 1 (colour saturation is directly proportional to the radiation intensity). Ellipses at the boundaries of the area indicate the location of the sensors. The contamination was exfoliated at a radiation intensity at the point of contamination of more than $50 \%$ compared to the maximum.

The cleaning was carried out in 4 cycles, which is how long the complete cleaning lasted with all the emitters on. At each step, a power factor was determined for each emitter, which was normalized to $[0,1]$. Table 3 shows the parameters obtained for each cleaning cycle, 
and the power determined by them. To take into account the presence of contamination in the liquid with each cycle, increase the number of suspended particles. For a better illustration, the input parameters $x_{1}$ and $x_{2}$ in the table are not normalized, and $\mathrm{u}$ is given the most normalized, because it is with such power factors that the processing takes place in the next cleaning cycle.

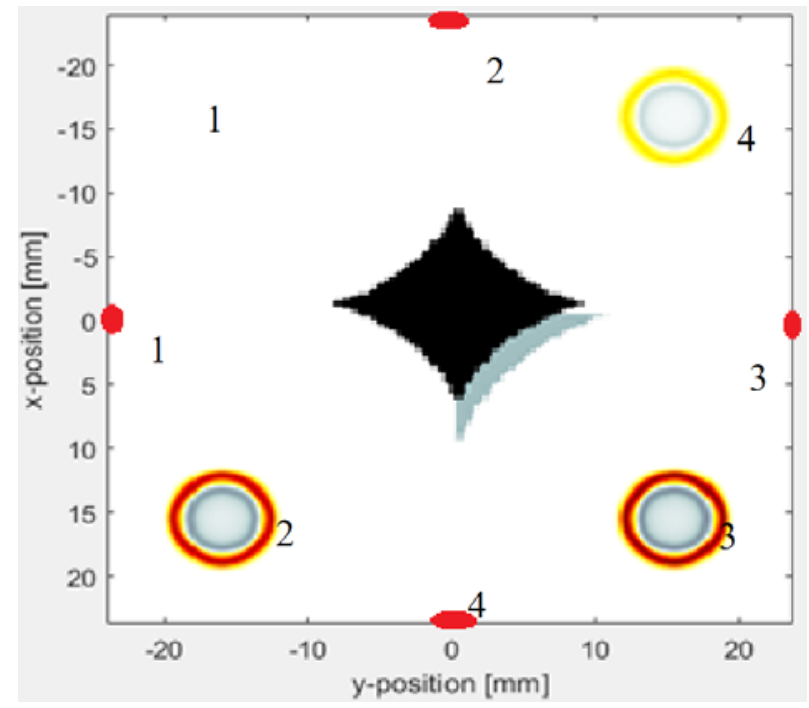

Fig. 6. Modelling of ultrasonic cleaning.

Table 3. Input and output parameters in ultrasonic cleaning.

\begin{tabular}{|c|c|c|c|c|}
\hline$\underset{\#}{\text { Step }}$ & $\mathbf{x}_{1}$ & $\mathbf{x}_{2}$ & $\mathbf{u}$ & $\begin{array}{c}\text { Power } \\
\text { saving, } \\
\%\end{array}$ \\
\hline 0 & - & - & {$\left[\begin{array}{llll}1 & 1 & 1 & 1\end{array}\right]$} & 0 \\
\hline 1 & $\begin{array}{c}{\left[\begin{array}{ll}0.0010 \\
0 & 0.0597 \\
0.0970]\end{array}\right.} \\
\end{array}$ & $\begin{array}{l}{\left[\begin{array}{lll}0.2240 & 0.0055 \\
0.0015 & -3.4094\end{array}\right]}\end{array}$ & $\begin{array}{c}{\left[\begin{array}{lll}0 & 0.9175 & 1.0000 \\
& 0.3638\end{array}\right]}\end{array}$ & 42.97 \\
\hline 2 & $\begin{array}{c}{[-0.0005} \\
00.0010 \\
0.0016]\end{array}$ & 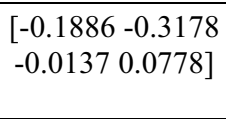 & 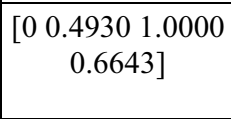 & 46.07 \\
\hline 3 & $\begin{array}{c}{\left[\begin{array}{ll}0 & 0\end{array}\right.} \\
0.00051 \\
0.00051]\end{array}$ & $\begin{array}{c}{\left[\begin{array}{lll}-0.0153 & 0.2671 \\
0.0510 & 0.0100\end{array}\right]}\end{array}$ & $\begin{array}{c}{\left[\begin{array}{lll}0 & 0.7260 & 1.0000 \\
0.1956\end{array}\right]} \\
\end{array}$ & 51.96 \\
\hline Total & & & $\begin{array}{c}\Sigma[13.13654 \\
2.2237]=10.3602\end{array}$ & 35.25 \\
\hline
\end{tabular}

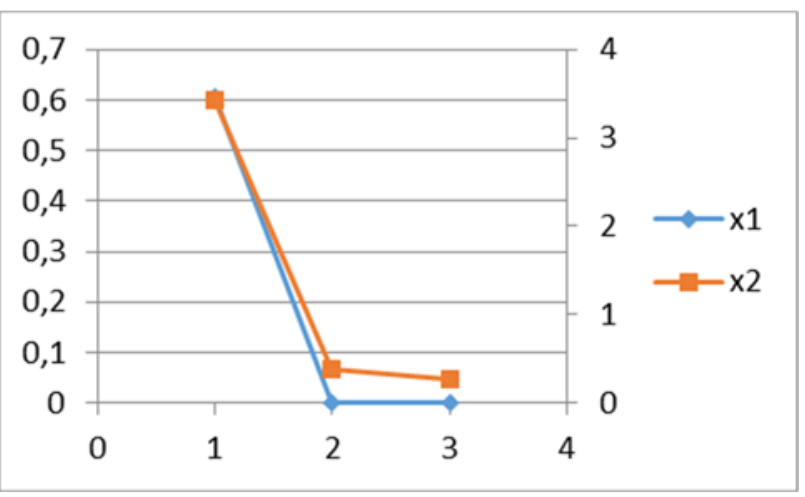

Fig. 7. Reduction of the deviation modules $x_{1}$ and $x_{2}$ by cleaning cycles.
These tables show that in each cycle, the maximum power factor of the emitter, which is closest to the contamination. At the same time, there is a constant decrease in the modulus of the vectors $\mathrm{x} 1$ and $\mathrm{x} 2$. A graphical representation of the reduction of input parameters is presented in Fig. 7.

One of the indicators of the need to stop the cleaning process is the acquisition of the limit value of the module for $x_{1}$ and $x_{2}$.

Due to the fact that the emitters did not work at full power, energy savings amounted to 35 . $25 \%(100 \%$ $10.3602 /(4 \cdot 4) \%)$.

\section{Conclusions}

In this work, a method of evaluating the course of the process of ultrasonic cleaning by ultrasonic responses was formed. To determine it, the purification of bodies of different configurations was modelled and the analysis of the received signals was performed. Parameters of responses that were considered during the analysis: the time of receipt of the threshold value of the signal and the first three harmonics. It was decided to limit the time of receipt of the signal threshold value and the nonlinearity coefficient of the second order to determine the assessment of the course of the ultrasonic cleaning process. It is the relative changes of these values and became the input parameters for the 3-D fuzzy interval controller, which performs spatially distributed control of the ultrasonic cleaning process. Energy savings using the established estimation technique and 3-D fuzzy interval controller amounted to about $35 \%$ compared to traditional control, which is limited to time only.

The authors express their sincere gratitude to Kryvyi Rih National University for support in conducting research.

\section{References}

1. W. Tangsopha, J. Thongsri, A Novel Ultrasonic Cleaning Tank Developed by Harmonic Response Analysis and Computational Fluid Dynamics. Metals. $\quad$ 10(3), $\quad 335 \quad$ (2020). doi:10.3390/met10030335

2. R. Roohia R., E. Abedib, S.M. Hashemi,K. Marszałek, J.M. Lorenzo, F. Barbae, Ultrasoundassisted bleaching: Mathematical and 3D computational fluid. Innovative Food Science and Emerging Technologies. 55, 66-79 (2019). doi:10.1016/j.ifset.2019.05.014

3. F. Duran, M. Teke, Design and implementation of an intelligent ultrasonic cleaning device. Intelligent Automation and Soft Computing (2018). doi: 10.31209/2018.11006161

4. A. Rahim, H. Bargoshadi, S. Sarrafi, Design and Manufacture an Ultrasonic Dispersion System. Sensors \& Transducers Journal. 126(3), 52-63 (2011). doi:10.1109/CSPA.2011.5759903

5. C. Rajani, A. Klami, A.Salmi, T. Rauhala, E.Haeggström, P. Myllymäki, Detecting industrial 
fouling by monotonicity during ultrasonic cleaning. in AALBORG:018 IEEE 28th International Workshop on Machine Learning for Signal Processing (MLSP), Aalborg, Denmark, 17-20 September, 2018. doi:10.1109/MLSP.2018.8517080

6. A. Simeone, E. Woolley, J. Escrig, N.J. Watson, Intelligent Industrial Cleaning: A Multi-Sensor Approach Utilising Machine Learning-Based Regression. Sensors. 20, $3642 \quad$ (2020). doi:10.3390/s20133642

7. V. Morkun, O. Kravchenko, Adaptive control over ultrasonic cleaning of mining equipment. $E 3 S \mathrm{Web}$ of Conferences (2020), 01005 (2020). doi:10.1051/e3sconf/202020101005

8. I. Papa, V. Lopresto, A. Langella, Ultrasonic inspection of composites materials: Application to detect. International Journal of Lightweight Materials and Manufacture. 4(1),37-42 (2021). doi:10.1016/j.ijlmm.2020.04.002

9. S. Majhi, A. Mukherjee, N. George, V. Karaganov, Corrosion Monitoring in Steel Bars using Laser Ultrasonic Guided Waves and Advanced Signal Processing. Mechanical Systems and Signal Processing. 149, $107176 \quad$ (2021). doi: 10.1016/j.ymssp.2020.107176

10. Z. Liao, X. Zhang, T. Liu, J. Jia, S.T. Tu, Characteristics of high-temperature equipment monitoring using dry-coupled ultrasonic waveguide transducers. Ultrasonics. 108, 106236 (2020). doi:10.1016/j.ultras.2020.106236

11. Z.-F. Yang, Y. Tian, H.- Q. Zhou, Y. Xu, W. - B. Zhang, J.- M. Li, Nonlinear Ultrasonic Response of TATB-Based Polymer, in 19th World Conference on Non-Destructive Testing 2016, 1-8 (2016).

12. Z. Yang, Y. Tian, W. Li, H.-Q. Zhou, W.-B. Zhang, J.- M. Li, Experimental Investigation of the Acoustic Nonlinear Behavior in Granular Polymer Bonded Explosives with Progressive Fatigue Damage. Materials. 10, 660 (2017). doi:10.3390/ma10060660

13. V. Morkun, N. Morkun, A. Pikilniak. The Propagation of Ultrasonic Waves in Gas-containing Suspensions, Cambridge Scholars Publishing, (2019), p 142.

14. B.E. Treeby, T. Cox, k-Wave: MATLAB toolbox for the simulation and reconstruction of photoacoustic wave fields. Journal of Biomedical Optics. 15(2), 021314 (2010). doi: 10.1117/1.3360308

15. B. E. Treeby, J. Jaros, A. P. Rendell, B. T. Cox, Modelling nonlinear ultrasound propagation in heterogeneous media with power law absorption using a k-space pseudospectral method. Acoustical Society of America. 131(6), 4324-4336 (2012). doi: $10.1121 / 1.4712021$

16. N. Karnik, J. Mendel, Centroid of a type-2 fuzzy set, Inform.Sci., 132, 195-220, (2001). 